\title{
Achieving Polio Eradication: A Need for Innovative Strategies
}

\author{
Basile Keugoung ${ }^{1,2 *}$, Richard Fotsing ${ }^{1}$, Bart Criel $^{3}$, Jean Macq ${ }^{4}$ \\ ${ }^{1}$ Ministry of Public Health, Yaounde, Cameroon; ${ }^{2}$ Education and Health Development Group, Dschang, Cameroon; ${ }^{3}$ Institute of Tro- \\ pical Medicine, Antwerp, Belgium; ${ }^{4}$ Institut de Recherche Santé et Société, Université Catholique de Louvain, Bruxelles, Belgique. \\ Email: *bkeugoung@itg.be
}

Received December $13^{\text {th }}, 2011$; revised January $17^{\text {th }}, 2012$; accepted January $29^{\text {th }}, 2012$

\begin{abstract}
Objective: The objective of this study was to analyze the current strategies used for eradicating wild polio viruses (WPV) and to propose some innovative strategies that may help to accelerate the progress towards polio eradication. Methods: We assessed the current strategies proposed by the World Health Organization, and the effectiveness of the current trivalent oral polio vaccine types 1, 2 and 3 (tOPV) schedule. Results: With the current schedule, tOPV is given four times to the child during his first year of life. After the four doses, $27 \%, 10 \%$ and $30 \%$ of children vaccinated are not immunized against WPV types 1, 2 and 3 respectively. In addition, low access to health care, insufficient funding of the routine immunization activities, and weak health systems hamper the tOPV coverage and the early detection of WPV cases for a rapid outbreak response. All these issues could explain the recurrence of WPV outbreaks, even in countries free of polio for many years. Therefore, we propose for countries of non-polio free regions, a new routine polio vaccination schedule composed of four doses of tOPV, followed by three doses of monovalent OPV type 1 , and lastly by three doses of bivalent OPV types 1 and 3 . With this schedule, of children fully vaccinated, 100\%, $90 \%$ and 99\% will be immunized against WPV types 1, 2 and 3 respectively. In addition, adequate funding for routine immunization activities and health system strengthening are proposed to accelerate the achievement of the polio eradication goal in a near future. Conclusions: The polio eradication goal is achievable. However, innovative strategies are urgently needed to improve the effectiveness and the efficiency of the routine polio immunization program.
\end{abstract}

Keywords: Polio Eradication; Vaccination; Wild Polio Virus; Immunization Program

\section{Introduction}

Significant efforts have been made so far along the path to polio eradication. After 23 years of Global polio eradication initiative, a lot of improvement has been made in terms of reduction of wild polio virus (WPV) cases from 350,000 estimated cases in 1988 to 1352 cases in 2010, DALYS and QUALYS earned and deaths averted [1]. Nigeria, the African country with the bulk of WPV cases in the past has made significant efforts, and has reduced WPV from 830 cases in 2005 to 21 cases in 2010. These global achievements are the results of the global partnership against polio, the improvement of the polio immunization coverage through routine vaccination and polio mass immunization campaigns, and of acute flaccid paralysis (AFP) surveillance.

With this significant reduction of WPV, there is increasing hope that the polio eradication goal can be achieved. The strategies used so far include routine vac-

*Corresponding author. cination using trivalent oral polio vaccine types 1,2 and 3 (tOPV) for children under one year of age, and supplementary polio immunization mass campaigns through local, national, or regional immunization days using either tOPV, bivalent OPV types 1 and 3 (bOPV 1.3) or monovalent OPV type 1 (mOPV 1 ).

In 2010, $12(60 \%)$ of the 20 countries that reported WPV cases were from sub-Saharan Africa. These countries notified 657 (49\%) of the 1352 WPV cases reported worldwide. Other countries that notified WPV cases were Afghanistan, Pakistan, Kazakhstan, Russian Federation, Tajikistan, Turkmenistan, Nepal and India [2]. With the resurgence of WPV cases in countries that had been free of polio for many years, it will be difficult to eradicate polio by the end of 2012 as planned, and innovative strategies are urgently needed to achieve this important health goal [3].

Therefore, we aim to identify the weaknesses of the strategies used so far, and to propose some innovative strategies to reinforce the battle against the WPV. This 
may help to accelerate the progress towards polio eradication in the coming years.

\section{Current Strategies Used for Eradicating Polio and Their Weaknesses}

Immunization programs consider children aged less than one year as the target group for routine vaccination. The objective of the polio administrative coverage is to reach at least $80 \%$. The schedule for polio vaccination recommends 4 doses of tOPV given at birth, 6, 10 and 14 weeks of age. In some countries, a recall dose of tOPV is recommended at 16 months of age. According to Grassly and colleagues [4], tOPV used in developing countries for routine polio immunization has a very low protective efficacy per dose- $11 \%$ for tOPV compared to $30 \%$ for the mOPV1. Also, 14 doses of tOPV compared to 5 doses of mOPV1 are required to protect $80 \%$ of children against WPV type 1 . In Northern Nigeria, with one dose of mOPV1, $67 \%$ of children were protected while only $16 \%$ of children were protected with one dose of tOPV [5]. In developing countries, after four doses of tOPV, $73 \%, 90 \%$ and $70 \%$ of children are immunized against WPV types 1, 2 and 3 respectively [6]. Consequently, with this routine polio immunization schedule, $27 \%, 10 \%$ and $30 \%$ of children fully vaccinated are not immunized against WPV types 1, 2 and 3 respectively, and they could carry and spread the WPV in the community [7]. The surveillance of polio cases is based on AFP cases detection followed by stool examination for WPV identification. Unfortunately, this AFP-based surveillance is not sensitive as only 0.1 to $1 \%$ of polio cases develop paralysis. In addition, the low access to health care, the false beliefs of the population concerning the child palsy, the poor quality of the health care, and the low involvement of private health providers, traditional healers and community leaders in AFP surveillance are others factors which hamper the early detection of WPV cases.

The actual WHO policy concerning polio eradication is to respond against each detected WPV case by at least two rounds of polio mass immunization campaigns either at sub-national, national or regional level. Before a WPV infected child with AFP is detected, the WPV have all the time to spread among non-immunized children. These infected non-immunized children could excrete WPV in their stools for more than two months. With the high mobility of population within and between countries mobile populations have also low access to immunization services, and the poor hygiene and sanitation, WPV could easily circulate even when polio mass immunization campaigns are organized. Neighboring countries of endemic or epidemic ones are therefore all at risk of hosting a dormant WPV circulation. Although these mass immunization campaigns may have a direct impact on polio immunization coverage, they are also known for their destructive effect on routine activities [8].

Finally, the weak health systems in developing countries have been also described as the major obstacle to the achievement of diseases control programs goals [9].

\section{Some Innovative Strategies}

In order to give a final boost to polio eradication, we suggest that the following policy issues and challenges should be taken into consideration by policymakers and Global health initiatives concerning immunization programs in polio endemic regions.

\subsection{Review of the Immunization Schedule for Children under One Year of Age}

The schedule of routine polio vaccination should optimize the immunization of children against the three types of the WPV. Therefore, we propose that at birth, 6, 10 and 14 weeks, tOPV should be given, followed at 18, 22 and 26 weeks by mOPV 1 , and then at 30,34 and 40 weeks by bOPV 1.3. Furthermore, at the end of each year, the number of children not immunized against polio during routine activities should be estimated to propose the best time for organizing supplementary polio immunization activities to maintain the herd immunity.

The advantages of this schedule are 1) the immunization of $100 \%, 90 \%$ and $99 \%$ of children fully vaccinated against WPV types 1,2 and 3 respectively. These protection rates are sufficient to maintain high immunity among children and eradicate polio (Table 1); 2) the monthly follow-up of the child during his first year of life; and

Table 1. Effectiveness of a routine polio vaccination schedule with four doses of tOPV, and three doses mOPV 1 and bOPV 1.3.

\begin{tabular}{|c|c|c|c|c|c|c|c|c|c|}
\hline \multirow[b]{2}{*}{ Vaccine type } & \multicolumn{3}{|c|}{ Wild polio virus type 1} & \multicolumn{3}{|c|}{ Wild polio virus type 2} & \multicolumn{3}{|c|}{ Wild polio virus type 3} \\
\hline & $\begin{array}{c}\text { Children } \\
\text { Vaccinated }\end{array}$ & $\begin{array}{l}\text { Children } \\
\text { immunized }\end{array}$ & $\begin{array}{c}\text { Children } \\
\mathrm{NI}^{\mathrm{a}}\end{array}$ & $\begin{array}{c}\text { Children } \\
\text { Vaccinated }\end{array}$ & $\begin{array}{c}\text { Children } \\
\text { Immunized }\end{array}$ & $\begin{array}{c}\text { Children } \\
\text { NI }\end{array}$ & $\begin{array}{c}\text { Children } \\
\text { Vaccinated }\end{array}$ & $\begin{array}{l}\text { Children } \\
\text { immunized }\end{array}$ & $\begin{array}{c}\text { Children } \\
\text { NI }\end{array}$ \\
\hline tOPV & $100^{\mathrm{b}}$ & 73 & 27 & 100 & 90 & 10 & 100 & 70 & 30 \\
\hline mOPV 1 & 27 & 26 & 1 & 10 & & 10 & 30 & & 30 \\
\hline bOPV 1.3 & 1 & 1 & 0 & 10 & & 10 & 30 & 29 & 1 \\
\hline Overall program effectiveness & 100 & 100 & 0 & 100 & 90 & 10 & 100 & 99 & 1 \\
\hline
\end{tabular}

${ }^{\mathrm{a}}$ Not immunized; ${ }^{\mathrm{b}}$ All the figures represent the number of children. 
lastly 3) the reduction of the number of polio mass immunization campaigns.

In fact, with the current schedule, after 14 weeks, the child is seen much later, at 36 weeks, for measles and yellow fever vaccination. This long delay leads to a high drop-out for immunization and other child programs such as growth monitoring, breast feeding and nutrients supplementation.

\subsection{Adequate Funding for the Routine Immunization Activities}

Routine immunization activities should be adequately financed so that health facilities can easily cover their target population. The Reach Every District (RED) approach based essentially on district immunization coverage achievement should move to a child-based approach. With this approach, activities should be carefully planned by health facilities and the required resources adequately allocated so that all children are vaccinate using the three types of OPV before their first anniversary.

\subsection{Regular Identification of the Target Population for Routine Immunization}

The target population should come from a local census of children in each village/quarter, done by local people (community health workers for example). The poor accuracy of demographic data and the lack of regular census has led Expanded Program of Immunization's managers to estimate the target population, with the proportion of children less than one year varying from one country to another (for example, $4 \%$ in Cameroon and $2.95 \%$ in Cote d'Ivoire). The advantage of this census is that it can provide accurate data for monitoring routine immunization activities.

\subsection{Change from the Reach Every District (RED) Approach to the Reach Every Child Approach}

The RED approach aims to achieve routine immunization coverage objectives in each district should be transformed into a Reach Every Child (REC) approach. The objective of the REC approach will be to vaccinate each child during routine activities. Therefore, barriers to vaccination should be regularly identified and addressed to improve access to immunization services in each basic administrative unit (village/quarter). Access to curative health care should be included in the immunization programs' strategies to improve the care of unhealthy children and more specifically of children with diseases preventable by immunization.

\subsection{Implication of All Stakeholders in AFP Surveillance and Health System Strengthening}

AFP surveillance should include all health providers es- pecially those of the private sector, traditional healers and the community health relays for a timely detection, investigation and reporting of AFP cases, and a timely response to WPV cases.

The strengthening of the health system by immunization programs is mandatory and should not be limited to the immunization activities but should take into consideration the whole health care delivery system. Indeed, vertical programs including immunization programs exert positive and negative effects on recipient health systems-the latter could jeopardize the whole health care delivery [10]. Therefore, the interface between the immunization program-as for all diseases control programs and the general health services should be regularly assessed to adapt both the program's policy and health services strategies in order to create synergies and optimize the performance of the immunization program and general health services.

\section{Conclusion}

The current strategies have had a high impact in WPV cases reduction. However, recurrent polio outbreaks in endemic and non-endemic countries including those that had been free of polio for many years have delayed progress towards eradication. Therefore, new and innovative strategies should be rapidly adopted, implemented and assessed for their scaling up. The adoption of a more effective routine polio immunization schedule should be the core content of these strategies.

\section{Acknowledgements}

We gratefully thank Kristof Decoster for his valuable inputs for editing this paper.

\section{REFERENCES}

[1] WHO, “Gobal Polio Eradication Initiative: History,” 2010. http://www.polioeradication.org/history.asp\#1988

[2] WHO, "Global Polio Eradication Initiative: AFP/Polio Case Count,” 2011.

http://apps.who.int/immunization_monitoring/en/diseases /poliomyelitis/afpextract.cfm

[3] Lancet, “Innovation for Polio Eradication,” Lancet Infectious Diseases, Vol. 11, 2011, p. 721. doi:10.1016/S1473-3099(11)70258-4

[4] N. C. Grassly, J. Wenger, S. Durrani, S. Bahl, J. M. Deshpande, R. W. Sutter, D. L. Heymann and R. B. Aylward, "Protective Efficacy of a Monovalent Oral Type 1 Poliovirus Vaccine: A Case-Control Study,” Lancet, Vol. 369, No. 9750, 2007, pp. 1356-1362. doi:10.1016/S0140-6736(07)60531-5

[5] H. E. Jenkins, R. B. Aylward, A. Gasasira, C. A. Donnelly, E. A. Abanida, T. Koleosho-Adelakan and N. C. Grassly, "Effectiveness of Immunization against Paralytic Polio- 
myelitis in Nigeria," The New England Journal of Medicine, Vol. 359, No. 16, 2008, pp. 1666-1674.

doi:10.1056/NEJMoa0803259

[6] S. Robertson, "Module 6: Poliomyelitis. The Immunological Basis for Immunization Series,” WHO, Geneva, 1993.

[7] Y. Berhane, C. J. Clements, J. M. Ndiaye and P. Taylor, "Has Routine Immunisation in Africa Become Endangered?” Lancet, Vol. 9, No. 11, 2009, pp. 655-656. doi:10.1016/S1473-3099(09)70268-3

[8] J. P. Unger, “Can Intensive Campaigns Dynamize Front Line Health Services? The Evaluation of an Immunization Campaigns in Thiès Health District, Senegal,” Social Sciences and Medicine, Vol. 32, No. 3, 1991, pp. 249-
259. doi:10.1016/0277-9536(91)90101-H

[9] A. Buve, S. Kalibala and J. McIntyre, "Stronger Health Systems for More Effective HIV/AIDS Prevention and Care," International Journal of Health Planning and Management; Vol. 18, No. S1, 2003, pp. S41-S51. doi:10.1002/hpm.725

[10] B. Keugoung, J. M. Macq, A. Buve, J. Meli and B. Criel, "The Interface between Health Systems and Vertical Programmes in Francophone Africa: The Managers' Perceptions," Tropical Medicine and International Health, Vol. 16, No. 4, 2011, pp. 478-485. doi:10.1111/j.1365-3156.2010.02716.x 